Check for updates

Cite this: New J. Chem., 2017, 41,3387

Received 4th January 2017 Accepted 22nd March 2017 DOI: 10.1039/c7nj00039a

rsc.li/njc

\section{Hybrid[4]arenes with anthracene units and tuneable cavities $\dagger$}

\author{
Tomasz Boinski and Agnieszka Szumna (DD *
}

\section{Introduction}

Macrocyclic skeletons composed of anthracene fragments expose large, hydrophobic, electron-rich panels into macrocyclic cavities. This feature greatly enhances complexation properties and additionally allows for easy detection of complexation events due to the inherent fluorescence of anthracene units. ${ }^{1}$ In comparison with other polyaromatic building blocks, anthracenes present a reasonable compromise between availability, chemical modifiability, structural rigidity and solubility. Therefore, over the years they have been widely used as building blocks for cyclophanes - hydrocarbons composed of aromatic units bridged by $n$-alkyl $(n \geq 2)$ chains. $^{2-6}$ More recently anthracene units were also used as parts of cageshaped anthraphanes, ${ }^{7}$ hybrid imidazolium-anthracene macrocycles $^{8,9}$ and shape-persistent tubes. ${ }^{10-15}$ These polyaromatic macrocycles effectively complex various fluorescent dyes, aromatic carbonyl derivatives ${ }^{14}$ and fullerenes. ${ }^{16}$ An oxidized form of anthracene - anthraquinone - has likewise been used for the construction of macrocyclic compounds using imine forming reactions. ${ }^{17,18}$ Quite intriguingly, anthracene-based building blocks have not been incorporated as parts of calixarenes macrocycles with aromatic panels linked by short methylene bridges. In this paper we present our approach towards synthesis of calixarene-type macrocycles using anthracene building blocks and towards post-synthetic modification of their cavity.

Institute of Organic Chemistry, Polish Academy of Science, Kasprzaka 44/52, 01-244 Warsaw, Poland. E-mail: agnieszka.szumna@icho.edu.pl

$\dagger$ Electronic supplementary information (ESI) available: NMR and ESI MS spectra and titration experiments. CCDC 1525253. For ESI and crystallographic data in CIF or other electronic format see DOI: 10.1039/c7nj00039a
Among the methods of synthesis of calixarene type hybrid macrocycles ${ }^{19-21}$ only two are reported to be reversible. The first one is based on the Friedel-Crafts reaction of dialkoxybenzyl bromides under dry conditions. ${ }^{22}$ The second one utilizes a TFA-catalyzed reaction between electron-rich aromatics and formaldehyde in chlorinated solvents at high temperatures. ${ }^{23-25}$ The reversibility of the macrocyclization reaction opens new synthetic possibilities as it allows for amplification of the thermodynamically most stable structures and allows for a template-type synthesis. Indeed, we recently demonstrated a high yielding, solvent-templated synthesis of pillar[5] $\operatorname{arenes}^{23}$ and synthesis of new calix[4]naphthalenes ${ }^{25}$ using this approach. Additionally, a dynamic covalent approach facilitated the synthesis of hybrid macrocycles consisting of different building blocks within a macrocyclic ring via a one-pot reaction. ${ }^{24}$ Recently, we further increased the dimensions of the building blocks aiming at the synthesis of anthracene-based macrocycles using this methodology. Herein, we report the structures and complexation properties of macrocycles obtained using such a method.

\section{Results and discussion}

Initially, we tested the possibility of obtaining homo-anthracene macrocycles, i.e. macrocycles consisting of identical anthracene building blocks. We used 1,4,5,8-tetramethoxyanthracene 2 and paraformaldehyde in TFA/DCE $(5 \% \mathrm{vol} / \mathrm{vol})$ at $80{ }^{\circ} \mathrm{C}$. We have previously reported that under such conditions the macrocyclization reaction of benzene analogs is reversible. ${ }^{24}$ In the case of 2 only undefined polymeric products were obtained, suggesting that homo-macrocycles are not thermodynamically preferred over 
Table 1 Optimization of synthesis of $\mathbf{3}$

\begin{tabular}{llccc}
\hline $\mathrm{C}_{1}=\mathrm{C}_{2}(\mathrm{M})$ & Solvent $^{a}$ & Time $(\mathrm{h})$ & TFA $(\%$ vol $/ \mathrm{vol})$ & Yield of $3(\%)$ \\
\hline 0.05 & DCE & 2 & 5 & 8 \\
0.05 & $\mathrm{DCE}$ & 4 & 5 & 10 \\
0.05 & $\mathrm{DCE}$ & 18 & 5 & 7 \\
0.05 & $\mathrm{DCE}$ & 4 & 2 & 3 \\
0.05 & $\mathrm{DCE}$ & 4 & 1 & 0 \\
0.05 & $\mathrm{DCE}$ & 4 & 15 & 10 \\
0.25 & $\mathrm{DCE}$ & 4 & 20 & 7 \\
0.05 & $\mathrm{DCE}$ & 4 & 30 & 4 \\
0.0125 & DCE & 2 & 1.25 & 5 \\
0.05 & $\mathrm{CHCl}_{3}$ & 4 & 5 & 9 \\
${ }^{a}$ All reactions & & &
\end{tabular}

polymeric products under current conditions. In the next step anthracene monomer 2 was combined with $\mathbf{1}$ (a building block based on a benzene ring) in a one pot reaction with formaldehyde in DCE catalysed by TFA. Two macrocyclic products 3 and 4 were isolated from the reaction mixture. Both products are hybrid[4]arenes that contain two anthracene-based units and two 1,3-dimethoxybenzene units, as was established based on NMR and mass spectra (calculated mass 1095.4295 for $\left[\mathrm{C}_{68} \mathrm{H}_{64} \mathrm{O}_{12} \mathrm{Na}\right]^{+}$, found 1095.4291). Optimization of the synthetic procedure (Table 1) resulted in the formation of 3 in 10\% yield. The yield of 3 is only slightly dependent on the initial concentration of substrates; however, it requires at least $5 \%$ of the catalyst and sufficient time for equilibration. These results are in agreement with the thermodynamic control of the reaction. In contrast, macrocyclic product $\mathbf{4}$ was obtained only in trace amounts, independent of the conditions, which may result from its lower thermodynamic stability.

${ }^{1} \mathrm{H}$ and ${ }^{13} \mathrm{C}$ NMR spectra of 3 reveal the presence of only $\frac{1}{4}$ of the maximum number of signals of the anthracene units and $\frac{1}{2}$ of the maximum number of signals of the benzene units (ESI $\dagger$ ). Methylene bridges are present as two doublets with a geminal coupling constant of $16.1 \mathrm{~Hz} .{ }^{1} \mathrm{H}^{-13} \mathrm{C}$ HSQC and ${ }^{1} \mathrm{H}^{-13} \mathrm{C}$ HMBC spectra indicate that methylene bridges link the macrocycle at the 2,6 positions of the anthracene unit and the 4,6 positions of the benzene unit. These characteristics suggest a $C_{2 \mathrm{~h}}$-symmetric structure for 3 with a two-fold axis passing through the centres of the anthracene units and a perpendicular mirror plane crossing the benzene units. In agreement with this symmetry, there are three signals from $\mathrm{CH}_{3}$ groups present in the spectrum. The signal at $4.00 \mathrm{ppm}$ was assigned to resorcinol methyl groups, more shielded signals at 3.74 and 3.54 were assigned to methyl groups from the anthracene building block positioned respectively meta and ortho in relation to the bridge. The modelled structure of 3 calculated by molecular mechanics is presented in Fig. $1 \mathrm{~b}$ and $\mathrm{c}$. The anthracene faces are positioned perpendicularly to the main macrocyclic plane (defined by four bridging methylene groups) and parallel to each other (distance between the planes is ca. $4.8 \AA$ ). According to this model, free rotation of the anthracene units along the connection points is hindered and consequently prohibits interconversion of the macrocyclic ring. In contrast, the benzene units can flip, but only by $180^{\circ}$. This model is in agreement with non-equivalency of protons at the methylene bridges (exhibiting geminal coupling due to hindered rotation of a)

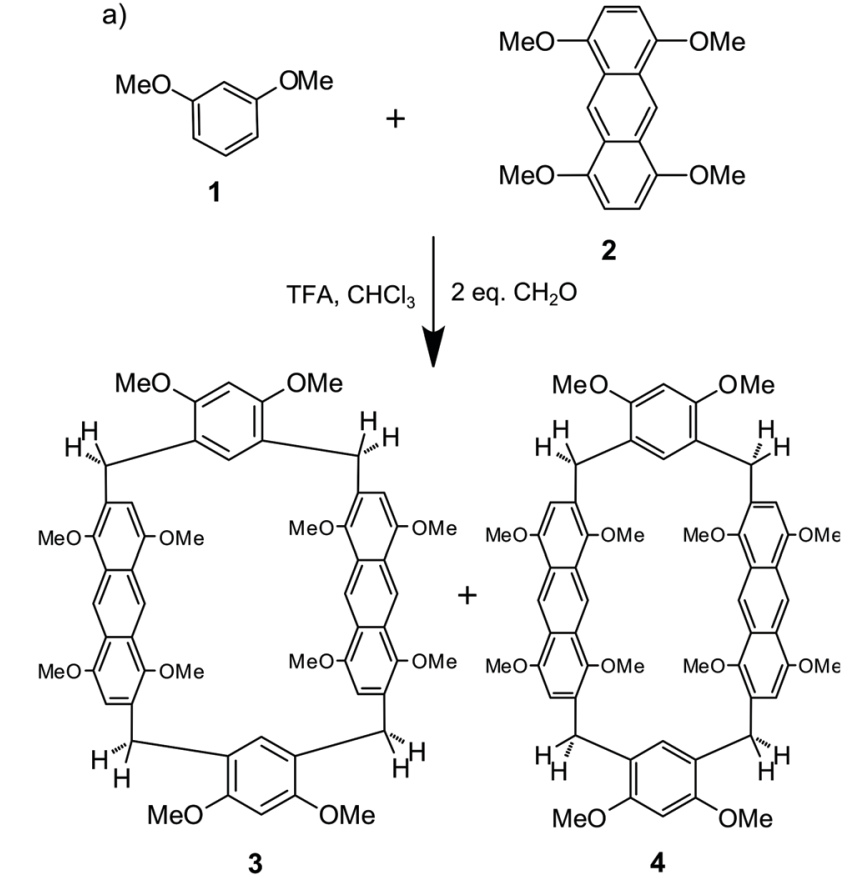

b)

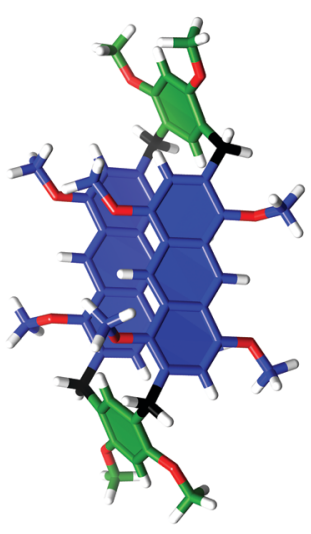

c)

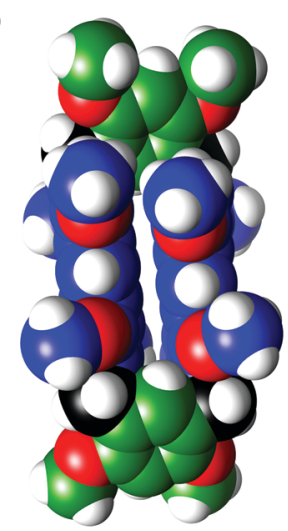

d)

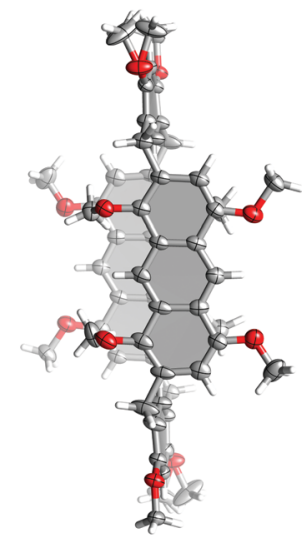

e)

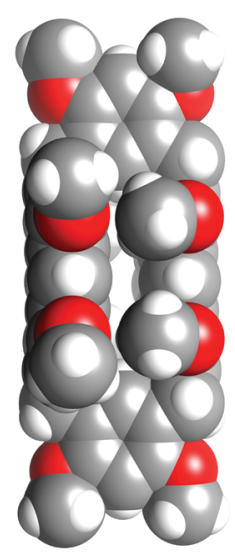

Fig. 1 (a) Synthesis of hybrid[4]arenes 3 and 4; (b) calculated structure of 3 (3D structure optimization module in ChemSketch); (c) van der Waals representation of the calculated structure of 3 ; (d) X-ray structure of 4 - ORTEP view (e) X-ray structure of $\mathbf{4}$ - van der Waals representation.

the anthracene units) and with considerable upfield shift of the signals of the methyl groups positioned at the anthracene units (due to close face-to-face proximity to these units). 
Hybrid[4]arene 4 was obtained in trace amounts and, due to purification problems, was not characterized in solution. However, we were able to obtain single crystals suitable for X-ray analysis from an enriched sample (Fig. 1d and e). In hybrid[4] arene 4 methylene bridges link the anthracene units at the 2,7 positions and the benzene units at the 4,6 positions. The molecule has a $C_{\mathrm{i}}$ crystallographic symmetry, but in the solution the symmetry of this molecule is expected to be higher $\left(C_{2 \mathrm{~h}}\right.$ with a two-fold axis passing through the centres of the benzene units and a perpendicular mirror plane crossing the anthracene units). The shape of the macrocyclic cavity is similar to that in the modelled structure of 3: two anthracene faces are parallel to each other and perpendicular to the main macrocyclic plane, while benzene rings are parallel to the main macrocyclic plane.

A comparison of UV and fluorescence spectra of hybrid[4]arene 3 and monomeric unit 2 indicates that the energies and intensities of the absorption bands (scaled to the same number of chromophores) do not change considerably (Fig. 2). However, for hybrid[4] arene 3, the quantum yield of emission increased, which may have originated from its rigid structure.

Due to parallel positions of anthracene faces, the cavity of macrocycle 3 is narrow. In order to tune the cavity's shape $\mathbf{3}$ was modified by the Diels-Alder reaction with benzyne (Fig. 3). Benzyne was in situ generated from benzene-diazonium-2carboxylate and reacted with 3 to yield 5 in $60 \%$ yield. The connection pattern and $C_{2 \mathrm{~h}}$ symmetry of $\mathbf{5}$ were confirmed by NMR. Molecular modeling indicates that the molecular cavity in 5 is expanded as compared with $\mathbf{3}$ (Fig. $3 \mathrm{~b}$ and c).

Macrocycles 3 and 5 were tested for complexation of rac-6, 7 and 8 (Fig. 4-6 and Table 2). The results indicate that both macrocycles interact with rac-6 and 7. For rac-6 the most affected proton during complexation is $\mathrm{H}_{\mathrm{c}}$ (downfield shift, Fig. 4), while for 7 the most affected proton is $\mathrm{H}_{\mathrm{a}}$ (upfield shift, Fig. 5). These differences suggest different modes of interactions for these two guests that may involve hydrogen bond-type interactions in the case of imidazolium based guest rac-6 (e.g. between the most acidic proton $\mathrm{H}_{\mathrm{c}}$ and ethereal oxygen atoms) and $\pi$-stacking interactions in the case of 7 (electron-poor pyridinium ring and electron-rich anthracene rings). ${ }^{1} \mathrm{H}$ NMR titrations indicate that the experimental points can be reasonably fitted only assuming simultaneous formation of $1: 1$ and $1: 2(\mathrm{H}: \mathrm{G})$.

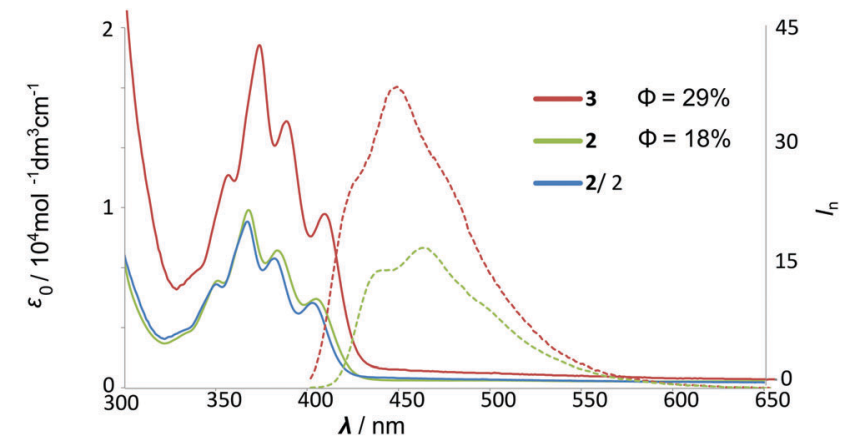

Fig. 2 UV-Vis and fluorescence spectra of monomer 2 and macrocycle $\mathbf{3}$ in chloroform (for the full-range spectrum see the ESI $\uparrow$ ). a)
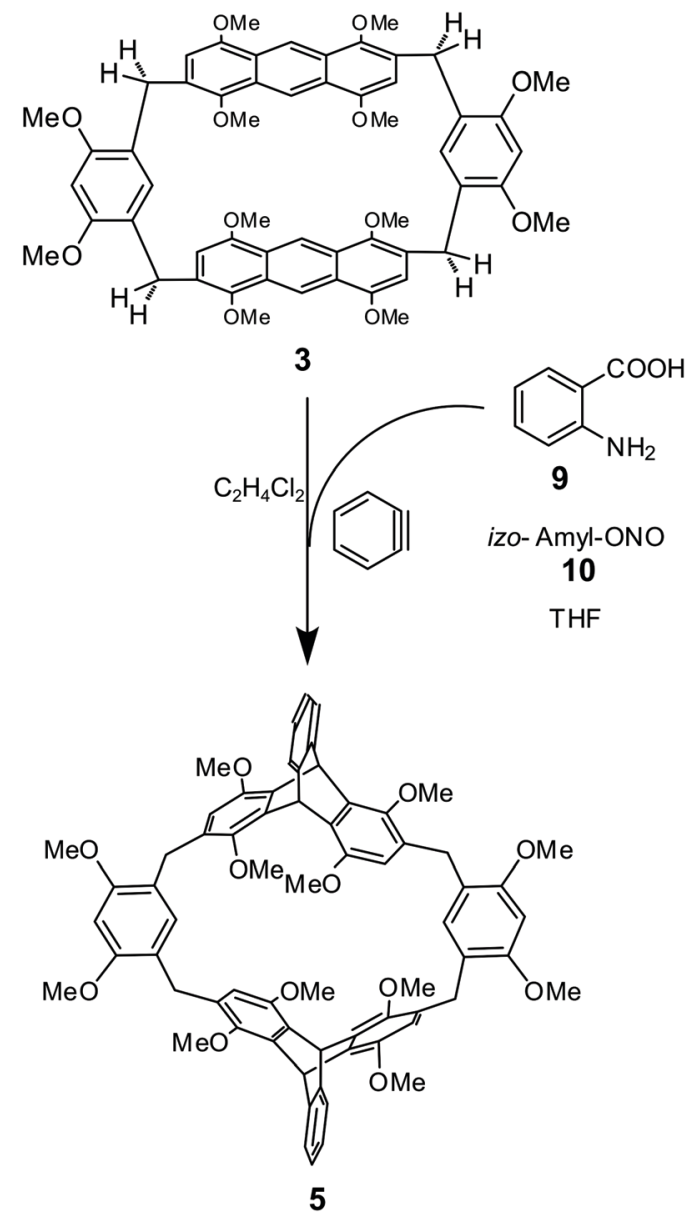

b)

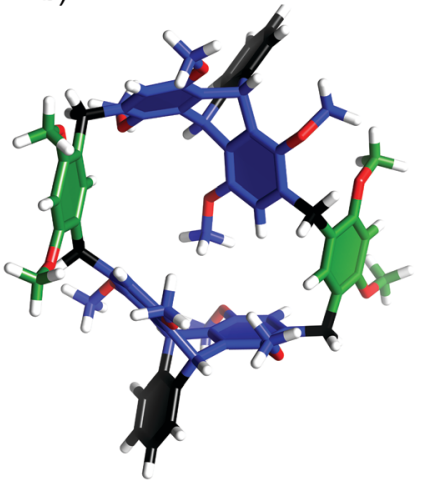

c)

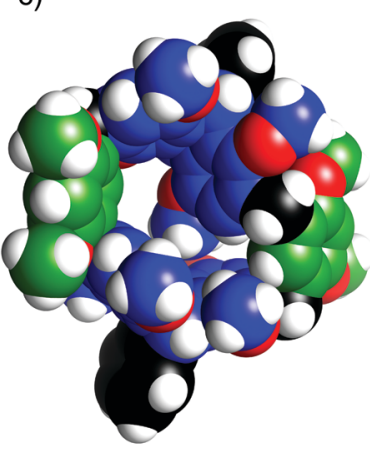

Fig. 3 (a) Modification of hybrid[4]arene 3 by the Diels-Alder reaction; (b) calculated structure of $\mathbf{5}$ (3D structure optimization module in ChemSketch); (c) van der Waals representation of the calculated structure of $\mathbf{5}$.

These qualitative and quantitative observations are consistent with the out-of-cavity complexation of guests rac- 6 and 7 by macrocycles 3 and 5. Complexation experiments using fluorescence monitoring were performed for $\mathbf{3}$; however, no effective changes were detected. A different complexation behavior was observed towards guest $\mathbf{8}$. Guest $\mathbf{8}$ is poorly soluble in chloroform and it remains virtually undissolved in the presence of macrocycle 3. However, in the presence of macrocycle $5(0.4 \mathrm{mM})$ 


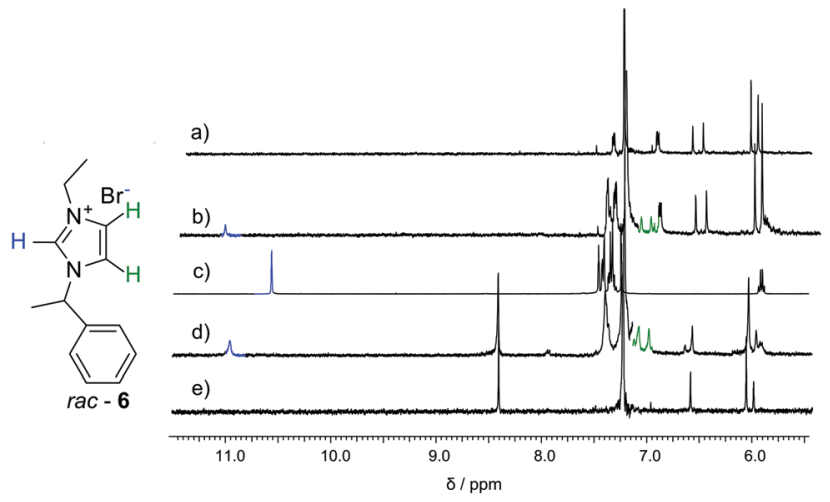

Fig. 4 NMR spectra of (a) $3(0.83 \mathrm{mM})$; (b) $3(0.83 \mathrm{mM})+$ rac- $6(0.95 \mathrm{mM})$; (c) rac-6 $(5.64 \mathrm{mM})$; (d) $5(0.59 \mathrm{mM})+$ rac-6 $(0.57 \mathrm{mM})$; (e) $5(0.59 \mathrm{mM})$, all in $\mathrm{CDCl}_{3}$ at $298 \mathrm{~K}$.

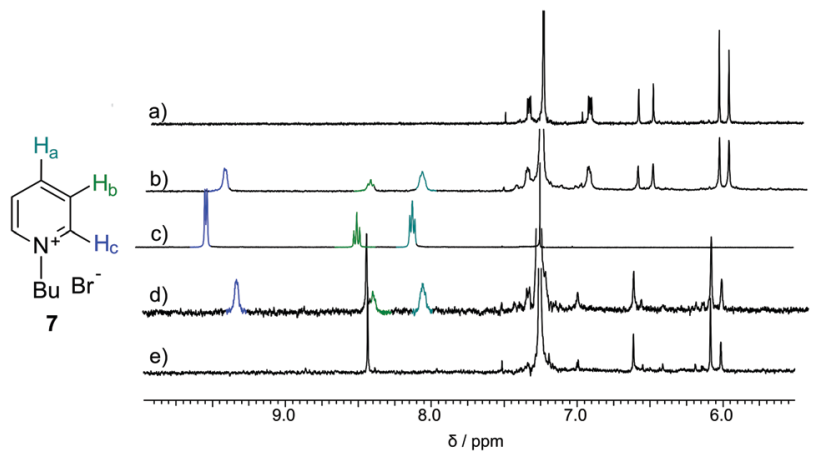

Fig. 5 NMR spectra of (a) $3(0.57 \mathrm{mM})$; (b) $3(0.57 \mathrm{mM})+7(0.51 \mathrm{mM})$; (c) 7 (19.57 mM); (d) $5(0.59 \mathrm{mM})+\mathbf{7}(0.86 \mathrm{mM})$; (e) $\mathbf{5}(0.59 \mathrm{mM})$ all in $\mathrm{CDCl}_{3}$

up to 2 equivalents of 8 can be dissolved in chloroform (Fig. 6a). All protons of guest $\mathbf{8}$ experience pronounced upfield shifts upon complexation. Molecular modelling indicates that the expanded cavity of 5 may accommodate a whole molecule of guest 8 (Fig. 6b).

In conclusion we have shown that an acid catalyzed condensation between 1,4,5,8-tetramethoxyanthracenes and formaldehyde leads to macrocyclic products only in the presence of additional benzenebased building blocks. This procedure results in the formation of anthracene-containing hybrid[4]arenes with a parallel arrangement of the anthracene units. The shape of the cavity in anthracene-based hybrid[4]arenes is tunable by the Diels-Alder reaction. The macrocycle with an expanded cavity is able to selectively complex pyridinium iodide in chloroform.

\section{Experimental}

3: To a solution of 2 (2.5 mmol, $0.745 \mathrm{~g}$ ) and 1 (2.5 mmol, $0.345 \mathrm{~g}$ ) in DCE $(50 \mathrm{ml})$ paraformaldehyde $(5 \mathrm{mmol}, 0.150 \mathrm{~g})$ and TFA $(2.5 \mathrm{ml})$ were added. The reaction was heated at reflux for $4 \mathrm{~h}$ under an argon atmosphere. During the reaction the color changed from yellow to dark green. The reaction was cooled to rt and an excess of $\mathrm{Na}_{2} \mathrm{CO}_{3}$ was added to neutralize TFA. The mixture was filtered off and evaporated to dryness. The product was isolated by chromatography (silica gel, $30 \mathrm{~g}, \mathrm{CHCl}_{3} /$ ethyl acetate from 100/0 to 100/7).

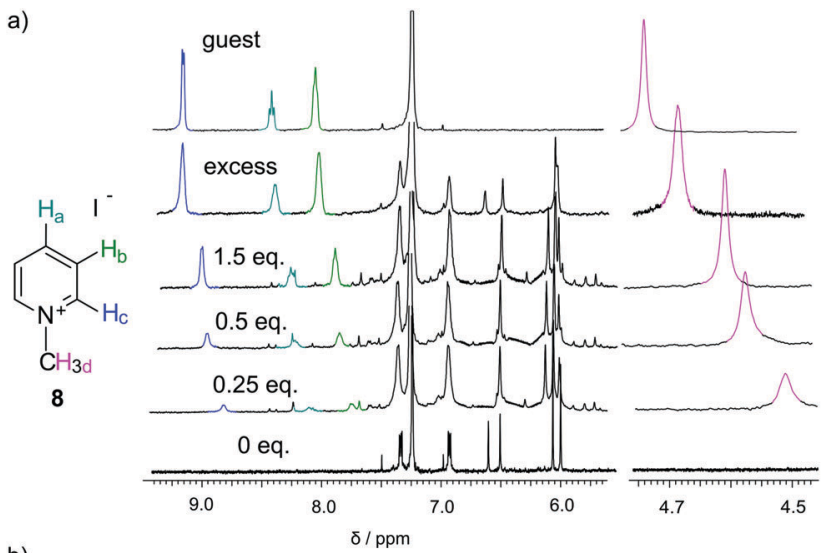

b)
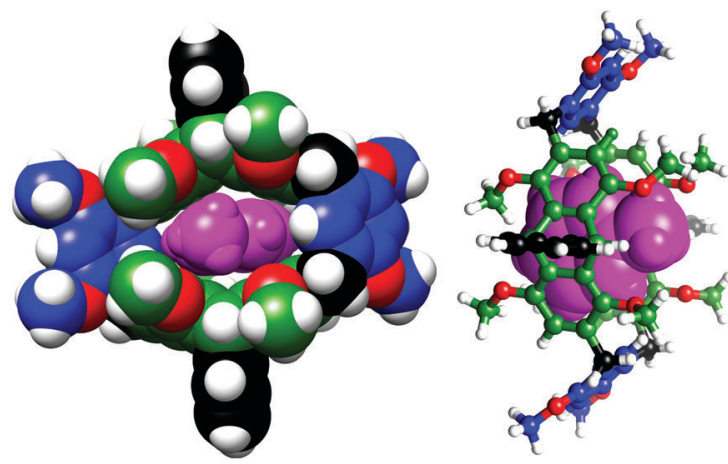

Fig. 6 (a) ${ }^{1} \mathrm{H}$ NMR spectra for complexation of methyl pyridinium iodate 8 by macrocyclic host $5\left(\mathrm{CDCl}_{3}, 298 \mathrm{~K}\right)$; (b) modelled structure of the complex (MM+ force field).

Table 2 Association constants determined by NMR titration (reported as $\beta$ values, $\left.K_{12}=\beta_{2} / \beta_{1}, \mathrm{CDCl}_{3}, 298 \mathrm{~K}\right)$

\begin{tabular}{llllll}
\hline & \multicolumn{2}{l}{ Guest rac-6 } & & & \multicolumn{2}{l}{ Guest 7 } \\
\cline { 2 - 5 } Host & $\log \left(\beta_{1}\right)$ & $\log \left(\beta_{2}\right)$ & & $\log \left(\beta_{1}\right)$ & $\log \left(\beta_{2}\right)$ \\
\hline 3 & 2.1 & 6.0 & 4.9 & 8.9 \\
5 & 2.9 & 6.3 & 3.3 & 8.4
\end{tabular}

Yield 10\% (115 mg). ${ }^{1} \mathrm{H}$ NMR ( $\left.\mathrm{CDCl}_{3}, 400 \mathrm{MHz}, 303 \mathrm{~K}\right): 8.44(2 \mathrm{H}, \mathrm{s})$, $6.62(1 \mathrm{H}, \mathrm{s}), 6.09(2 \mathrm{H}, \mathrm{s}), 6.02(2 \mathrm{H}, \mathrm{s}), 4.20\left(2 \mathrm{H}, \mathrm{d}, J^{2}=16.1 \mathrm{~Hz}\right), 4.00$ $(6 \mathrm{H}, \mathrm{s}), 3.83\left(2 \mathrm{H}, \mathrm{d}, J^{2}=16.1 \mathrm{~Hz}\right), 3.74(6 \mathrm{H}, \mathrm{s}), 3.54(6 \mathrm{H}, \mathrm{s}) .{ }^{13} \mathrm{C} \mathrm{NMR}$ $\left(\mathrm{CDCl}_{3}, 125 \mathrm{MHz}, 303 \mathrm{~K}\right):$ 156.3, 151.7, 147.9, 130.2, 125.4, 124.8, 121.1, 114.8, 104.5, 61.9, 55.8, 55.2, 28.3. HR MS (ESI) calcd for $\mathrm{C}_{56} \mathrm{H}_{56} \mathrm{O}_{12} \mathrm{Na}\left([\mathrm{M}+\mathrm{Na}]^{+}\right)$943.3669; found: 943.3665 .

\section{Benzenediazonium-2-carboxylate}

Anthranilic acid 9 (1 mmol, $137 \mathrm{mg})$ and trichloroacetic acid $(15 \mu \mathrm{l})$ were stirred in dry THF $(15 \mathrm{ml})$ under argon in a $25 \mathrm{ml}$ flask. To this solution iso-amyl nitrite $10(0.22 \mathrm{ml}, 1.7 \mathrm{mmol})$ was added over a period of 1-2 minutes. A mildly exothermic reaction occurred and the reaction mixture was stirred for a further 1.5 hours maintained at $18-25{ }^{\circ} \mathrm{C}$. A transient orange to brick-red precipitate appeared which was slowly converted to the tan product. Then the mixture was cooled to $10{ }^{\circ} \mathrm{C}$ and the product was collected by filtration on a plastic funnel and washed on the funnel with cold dry THF until the washings 
were colourless (Caution! The filter cake should not be allowed to become dry). Benzene-diazonium-2-carboxylate was then washed with two portions of 1,2-dichloroethane (2.5 ml each) to displace THF and the solvent-wet material was used in the next step.

5: A solution of $3(0.1 \mathrm{mmol}, 92 \mathrm{mg})$ in DCE $(25 \mathrm{ml})$ was prepared in a two necked $50 \mathrm{ml}$ flask equipped with a condenser and a septum under an argon atmosphere. The solution was heated to reflux. The solvent-wet benzenediazonium-2carboxylate was suspended in DCE $(10 \mathrm{ml})$ and the freshly prepared slurry was slowly added to the reaction mixture over $45 \mathrm{~min}$. The reaction was kept under reflux overnight. Then the solvent was removed by evaporation and the resulting solid was subjected to chromatography (silica gel, $5 \mathrm{~g}, \mathrm{CHCl}_{3} /$ ethyl acetate from $100 / 0$ to $100 / 3)$. Yield $60 \%$ (64 mg). ${ }^{1} \mathrm{H} \mathrm{NMR}\left(\mathrm{CDCl}_{3}, 400 \mathrm{MHz}\right.$, $303 \mathrm{~K}): 7.33\left(\mathrm{dd}, 4 \mathrm{H}, J^{3}=5.4 \mathrm{~Hz}, J^{4}=3.2 \mathrm{~Hz}\right.$ ), 6.95 (dd, $4 \mathrm{H}$, $\left.J^{3}=5.4 \mathrm{~Hz}, J^{4}=3.2 \mathrm{~Hz}\right), 6.62(\mathrm{~s}, 2 \mathrm{H}), 6.52(\mathrm{~s}, 2 \mathrm{H}), 6.07(\mathrm{~s}, 4 \mathrm{H}), 6.00$ $(\mathrm{s}, 4 \mathrm{H}), 3.93\left(\mathrm{~d}, 4 \mathrm{H}, J^{2}=16.6 \mathrm{~Hz}\right), 3.81(\mathrm{~s}, 12 \mathrm{H}) 3.66(\mathrm{~d}, 4 \mathrm{H}$, $\left.J^{2}=16.6 \mathrm{~Hz}\right) 3.63(\mathrm{~s}, 12 \mathrm{H}), 3.55(\mathrm{~s}, 12 \mathrm{H}) .{ }^{13} \mathrm{C} \mathrm{NMR}\left(\mathrm{CDCl}_{3}, 100 \mathrm{MHz}\right.$, $303 \mathrm{~K}): 156.7,150.5,147.5,146.0,139.5,132.5,131.7,131.2,124.9$, 123.7, 120.0, 110.0, 95.4, 61.7, 55.8, 55.7, 41.6, 29.3. HR MS (ESI) calcd for $\mathrm{C}_{64} \mathrm{H}_{64} \mathrm{O}_{12} \mathrm{Na}\left([\mathrm{M}+\mathrm{Na}]^{+}\right)$1095.4295; found: 1095.4291 .

\section{Crystal data for 4}

$\mathrm{C}_{57} \mathrm{H}_{57} \mathrm{Cl}_{3} \mathrm{O}_{12}\left(4 \times \mathrm{CHCl}_{3}\right), M=1040.37$, space group $C 2 / c$, $V=5099.6(10) \AA^{3}, Z=4, D_{\mathrm{c}}=1.355 \mathrm{~g} \mathrm{~cm}^{-3}, F_{000}=2184, \mathrm{CuK}_{\alpha}$ radiation, $\lambda=1.54184 \AA, T=173(2) \mathrm{K}, 2 \theta_{\max }=143.8^{\circ}, 8910$ reflections collected, 4904 unique $\left(R_{\text {int }}=0.0208\right)$. Final GooF $=$ 1.345, $R_{1}=0.1234, \mathrm{w} R_{2}=0.3838, R$ indices based on 2501 reflections with $I>2 \sigma(I)$ (refinement on $F^{2}$ ), 360 parameters, 18 restraints. Lp and absorption corrections applied, $\mu=2.159 \mathrm{~mm}^{-1}$. CCDC 1525253 contains the supplementary crystallographic data for this paper.

\section{Acknowledgements}

This work was supported by the National Science Center (grant 2013/09/N/ST5/00907).

\section{Notes and references}

1 M. Yoshizawa and J. K. Klosterman, Chem. Soc. Rev., 2014, 43, 1885.

2 B. Slepetz and M. Kertesz, J. Am. Chem. Soc., 2013, 135, 13720.
3 S. R. Jezowski, L. Zhu, Y. Wang, A. P. Rice, G. W. Scott, C. J. Bardeen and E. L. Chronister, J. Am. Chem. Soc., 2012, 134, 7459.

4 R. Kutsumizu, H. Shinmori and T. Takeuchi, Tetrahedron Lett., 2007, 48, 3225.

5 Y. Tobe, N. Utsumi, S. Saiki and K. Naemura, J. Org. Chem., 1994, 59, 5516.

6 A. Iwama, T. Toyoda, M. Yoshida, T. Otsubo, Y. Sakata and S. Misumi, Bull. Chem. Soc. Jpn., 1978, 51, 2988.

7 M. Servalli, N. Trapp, M. Worle and F. G. Klarner, J. Org. Chem., 2016, 81, 2572.

8 N. Ahmed, B. Shirinfar, I. Geronimo and K. S. Kim, Org. Lett., 2011, 13, 5476.

9 N. Ahmed, B. Shirinfar, I. S. Youn, A. Bist, V. Suresh and K. S. Kim, Chem. Commun., 2012, 48, 2662.

10 K. Hagiwara, M. Otsuki, M. Akita and M. Yoshizawa, Chem. Commun., 2015, 51, 10451.

11 K. Hagiwara, M. Akita and M. Yoshizawa, Chem. Sci., 2015, 6, 259.

12 Z. Li, Y. Sei, M. Akita and M. Yoshizawa, Chem. - Asian J., 2014, 9, 1016.

13 K. Yazaki, Y. Sei, M. Akita and M. Yoshizawa, Nat. Commun., 2014, 5, 5179.

14 K. Yazaki, N. Kishi, M. Akita and M. Yoshizawa, Chem. Commun., 2013, 49, 1630.

15 K. Hagiwara, Y. Sei, M. Akita and M. Yoshizawa, Chem. Commun., 2012, 48, 7678.

16 N. Kishi, M. Akita, M. Kamiya, S. Hayashi, H. F. Hsu and M. Yoshizawa, J. Am. Chem. Soc., 2013, 135, 12976.

17 Y. Okazawa, K. Kondo, M. Akita and M. Yoshizawa, J. Am. Chem. Soc., 2005, 127, 16404.

18 T. Ide, D. Takeuchi, K. Osakada, T. Sato and M. Higuchi, J. Org. Chem., 2011, 76, 9504.

19 B. Gao, L. L. Tan, N. Song, K. Li and Y. W. Yang, Chem. Commun., 2016, 52, 5804.

20 B. Hua, L. Shao, J. Zhou and G. Yu, New J. Chem., 2016, 40, 4756.

21 J. Zhou, J. Yang, B. Hua, L. Shao, Z. Zhang and G. Yu, Chem. Commun., 2016, 52, 1622.

22 M. Holler, N. Allenbach, J. Sonet and J. F. Nierengarten, Chem. Commun., 2012, 48, 2576.

23 T. Boinski and A. Szumna, Tetrahedron, 2012, 68, 9419.

24 T. Boinski, A. Cieszkowski, B. Rosa and A. Szumna, J. Org. Chem., 2015, 80, 3488.

25 T. Boinski, A. Cieszkowski, B. Rosa, B. Leśniewska and A. Szumna, New J. Chem., 2016, 40, 8892. 